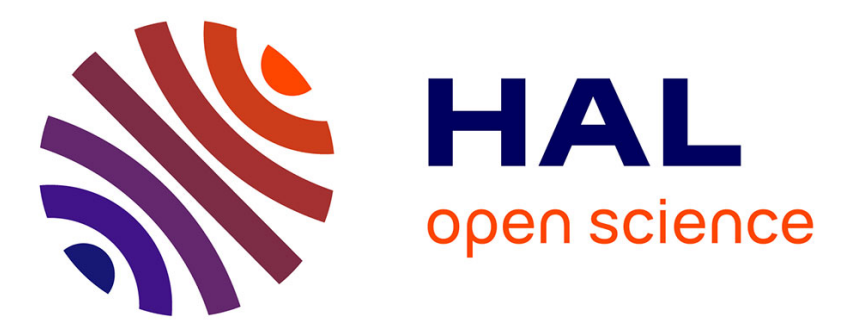

\title{
Enhancing light absorption in a nanovolume with a nanoantenna: theory and figure of merit
}

Emilie Sakat, Léo Wojszvzyk, Jean-Jacques Greffet, Jean-Paul Hugonin, Christophe Sauvan

\section{- To cite this version:}

Emilie Sakat, Léo Wojszvzyk, Jean-Jacques Greffet, Jean-Paul Hugonin, Christophe Sauvan. Enhancing light absorption in a nanovolume with a nanoantenna: theory and figure of merit. ACS photonics, 2020, 7 (6), pp.1523-1528. 10.1021/acsphotonics.0c00329 . hal-03036386

\section{HAL Id: hal-03036386 https://hal.science/hal-03036386}

Submitted on 2 Dec 2020

HAL is a multi-disciplinary open access archive for the deposit and dissemination of scientific research documents, whether they are published or not. The documents may come from teaching and research institutions in France or abroad, or from public or private research centers.
L'archive ouverte pluridisciplinaire HAL, est destinée au dépôt et à la diffusion de documents scientifiques de niveau recherche, publiés ou non, émanant des établissements d'enseignement et de recherche français ou étrangers, des laboratoires publics ou privés. 


\title{
Enhancing light absorption in a nanovolume with a nanoantenna: theory and figure of merit
}

\author{
Emilie Sakat, ${ }^{*}, \dagger, \ddagger$ Léo Wojszvzyk, ${ }^{\dagger}$ Jean-Jacques Greffet, ${ }^{\dagger}$ Jean-Paul Hugonin $^{\dagger}$ \\ and Christophe Sauvan*, $\dagger$ \\ $\dagger$ Universite Paris-Saclay, Institut d'Optique Graduate School, CNRS, Laboratoire Charles \\ Fabry, 91127, Palaiseau, France \\ $\ddagger$ Universite Paris Saclay, Center for Nanoscience and Nanotechnology, C2N UMR 9001, \\ CNRS, 91120 Palaiseau, France
}

E-mail: emilie.sakat@c2n.upsaclay.fr; christophe.sauvan@institutoptique.fr

\begin{abstract}
We study light absorption by a dipolar absorber in a given environment, which can be a nanoantenna or any complex inhomogeneous medium. From first-principle calculations, we derive an upper bound for the absorption, which decouples the impact of the environment from the one of the absorber. Since it is an intrinsic characteristic of the environment regardless of the absorber, it provides a good figure of merit to compare the ability of different systems to enhance absorption. We show that, in the scalar approximation, the relevant parameter is not the field enhancement but the ratio between the field enhancement and the local density of states. Consequently, a plasmonic structure supporting hot spots is not necessarily the best choice to enhance absorption. We also show that our theoretical results can be applied beyond the scalar approximation and the plane-wave illumination.
\end{abstract}




\section{Keywords}

Nanophotonics, Plasmonics, Nanoantenna, Absorption, Local Density of Optical States

Light absorption and emission are two fundamental processes of light-matter interaction $^{1,2}$. Absorption converts electromagnetic energy carried by photons to internal energy of matter carried by electrons or phonons. Controlling the absorption in small volumes is a major issue for numerous applications, such as photovoltaics ${ }^{3-5}$, sensing by surface-enhanced infrared absorption (SEIRA) ${ }^{6-8}$ or enhanced infrared photoexpansion nanospectroscopy ${ }^{9}$, thermal emission ${ }^{10,11}$, photothermal effects at the nanoscale for enhanced photocatalysis and nano-chemistry ${ }^{12-14}$.

Absorption inside subwavelength objects is weak but different strategies can be used to circumvent this limitation. The absorber properties, the illumination, or the environment can be engineered. Many works have been dedicated to the optimization of the absorption by a single nanoparticle ${ }^{15-21}$. For an absorber in a homogeneous medium, the physical bounds of the problem are known; they depend on the multipolar character of the particle. The maximum absorption cross-section of a molecule or a nanoparticle that behaves like a pure electric dipole (dipolar approximation), is $3 \lambda^{2} /\left(8 \pi n^{2}\right)$, with $\lambda$ the wavelength and $n$ the surrounding refractive index ${ }^{15,18}$. This upper bound can only be reached if the absorber polarizability $\alpha$ matches a precise value, $\alpha_{\mathrm{opt}}=i 3 \lambda^{3} /\left(8 \pi^{2} n\right)$. Subwavelength particles that go beyond the dipolar approximation offer additional degrees of freedom and are governed by different physical bound ${ }^{16-21}$. Absorption by an ensemble of particles in a homogeneous medium is yet another related problem with a different upper limit ${ }^{22}$.

Plunging the absorber in a complex medium or modifying the illumination offers additional possibilities to tailor the absorption ${ }^{10,23,24}$. In this work, we consider a subwavelength absorber in a complex environment (inhomogeneous or not) as depicted in Fig. 1. We focus on the absorption inside the absorber and do not discuss dissipation in the surroundings, if any. To fully exploit the control possibilities offered by the environment, it is crucial to know what is the relevant figure of merit. The absorption density is proportional to the local 
electric-field intensity $|\mathbf{E}(\mathbf{r})|^{225}$, which results from the environment and the absorber. It is highly desirable to decouple both contributions to provide a figure of merit that is intrinsic to the environment. Only then can we properly compare the ability of different structures to modify absorption.

Let us draw a parallel with spontaneous emission. In a complex medium, it is modified by a factor proportional to the photonic local density of states (LDOS ${ }^{26}$. For an emitter coupled to a resonant cavity, the emission enhancement has a well-known upper bound, the Purcell factor, which is intrinsic to the resonator and independent of the emitter ${ }^{27-29}$. The upper bound is reached if emitter and resonator fulfill a few matching conditions - spectral, spatial, and in polarization ${ }^{29}$.

The link between the absorption and the environment properties is more complex than the proportionality relation between emission and LDOS. Let us start by the simple case where the absorber perturbs only slightly the electromagnetic field. In this perturbation regime, the Born approximation can be used and the field inside the absorber is asssumed to be equal to the field $\mathbf{E}_{b}$ provided by the environment alone, see Fig. 1b. In that case, the absorption is simply proportional to the field enhancement $\left|\mathbf{E}_{b}\right|^{2} /\left|\mathbf{E}_{\text {inc }}\right|^{2}$. Hence, hot spots are often thought to provide large absorption enhancements and nanoantennas are often engineered for maximizing the field enhancement they provide ${ }^{30,31}$. However, if the presence of the absorber significantly affects the local field, a perturbative treatment is not valid
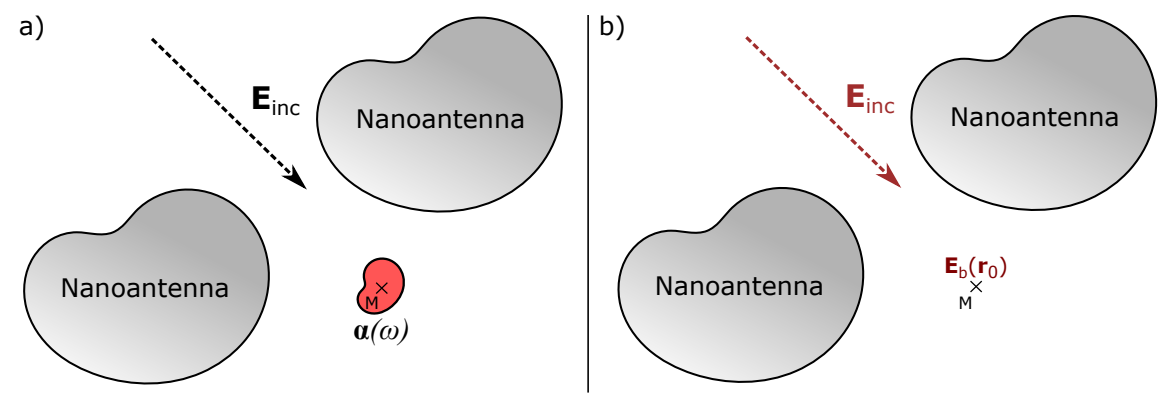

FIG. 1: (a) Subwavelength absorber (in red) in an arbitrary environment (in gray, referred to as "nanoantenna") illuminated by an incident field $\mathbf{E}_{\text {inc }}$. We focus on the absorption inside the particle. (b) Same problem without the absorber. The field $\mathbf{E}_{b}$ is the field scattered by the environment alone. 
and the situation is more complex: the field enhancement is not the only electromagnetic property of the environment that drives the absorption. For instance, it has been recently shown that, in a scalar quasi-static case, the absorption cross-section is inversely proportional to the square of the $\operatorname{LDOS}^{23}$. The work in Ref. ${ }^{23}$ considers absorption in a given system absorber + environment but does not decouple the contribution of the absorber from the impact of the environment. It does not study the physical bounds of the problem. Until now, no upper bound - kind of Purcell factor analogue - has been derived for the problem of an absorber in a complex environment.

Antenna theory provides a solution in one specific case. For an antenna receiving a signal from the direction $(\theta, \phi)$, the maximum absorption cross-section of the load is $G_{a}(\theta, \phi) \lambda^{2} /\left(4 \pi n^{2}\right)$, with $G_{a}$ the antenna gain ${ }^{32-34}$. This upper bound is reached if the load is impedance-matched with the antenna. The gain is defined for an emitting antenna as the fraction of the wall-plug power radiated in the direction $(\theta, \phi)$. Unfortunately, the $G_{a} \lambda^{2} /\left(4 \pi n^{2}\right)$ limit only applies to plane-wave illumination and to antennas working in the scalar approximation when one component of the electromagnetic field is dominant. In cases where the vectorial nature of the electromagnetic field cannot be neglected, the problem remains open. Moreover, the antenna point of view highlights the gain, whereas other derivations underline the field enhancement and the $\operatorname{LDOS}^{23}$. It is thus important to generalize existing results, while enlightening the link between them.

In this work, we derive a general upper bound for the power dissipated in a subwavelength absorber surrounded by any complex inhomogeneous environment. Owing to its subwavelength dimensions, the absorber is treated in the electric-dipole approximation. On the other hand, the electromagnetic properties of the environment are treated rigorously without any approximation. The upper bound is independent of the absorber; it entirely depends on the environment and the illumination. Thus, it provides a relevant figure of merit for comparing the ability of different systems to enhance absorption. This figure of merit results from the interplay between two electromagnetic properties of the environment, the field enhancement 
and the Green tensor. We show under which assumptions the gain is a relevant parameter. We finally discuss under which conditions the system can reach the upper bound. We apply the theory to a few emblematic examples of nanophotonics: a plasmonic dimer nanoresonator, dielectric nanoantennas, and a silicon-on-insulator (SOI) ridge waveguide. We evidence that a plasmonic system providing extremely large field enhancements is not necessarily an optimal choice to increase absorption.

We consider the problem illustrated in Fig. 1(a). An absorbing subwavelength particle (in red) is placed in a complex absorbing or non-absorbing environment (in gray) and illuminated by an incident field $\mathbf{E}_{\text {inc }}$. We focus on the absorption inside the particle. We consider passive materials and use the $\exp (-i \omega t)$ convention for time-harmonic fields, with $\omega=2 \pi c / \lambda$. The following derivations apply to any incident field and any environment geometry. We refer to the environment as "the antenna", but no particular assumption is made on its geometry.

The total electric field can be written as $\mathbf{E}(\mathbf{r}) \equiv \mathbf{E}_{b}(\mathbf{r})+\mathbf{E}_{s}(\mathbf{r})$, where $\mathbf{E}_{b}$ is the field in the absence of the absorber, see Fig. 1(b), and $\mathbf{E}_{s}$ is the field scattered by the absorber. It is the field radiated by the current source induced inside the absorber by the exciting field. We now make the sole assumption of our derivation. We assume that the subwavelength particle scatters light like an electric dipole $\mathbf{p}$ located at $\mathbf{r}=\mathbf{r}_{0}$. The scattered field is then $\mathbf{E}_{s}(\mathbf{r})=\mu_{0} \omega^{2} \mathbf{G}\left(\mathbf{r}, \mathbf{r}_{0}\right) \mathbf{p}$, with $\mathbf{G}$ the Green tensor of the antenna alone.

The power dissipated in the particle is the difference between extinction and scattering, $P_{a}=P_{e}-P_{s}{ }^{25}$. Within the dipole approximation, $P_{e}=-\frac{1}{2} \omega \operatorname{Im}\left(\mathbf{p}^{\dagger} \mathbf{E}_{b}\right)$ and $P_{s}=\omega \mathbf{p}^{\dagger} \mathbf{g p}$, with $\mathbf{g} \equiv \frac{1}{2} \mu_{0} \omega^{2} \operatorname{Im}\left[\mathbf{G}\left(\mathbf{r}_{0}, \mathbf{r}_{0}\right)\right]$ (see Supporting Information). It follows

$$
P_{a}=-\frac{1}{2} \omega \operatorname{Im}\left(\mathbf{p}^{\dagger} \mathbf{E}_{b}\right)-\omega \mathbf{p}^{\dagger} \mathbf{g} \mathbf{p}
$$

where $\mathbf{E}_{b} \equiv \mathbf{E}_{b}\left(\mathbf{r}_{0}\right)$ and $\mathbf{p}^{\dagger}$ is the conjugate transpose of $\mathbf{p}$. The induced dipole is $\mathbf{p}=$ $\varepsilon_{0} \boldsymbol{\alpha}(\omega)\left[\mathbf{E}_{b}\left(\mathbf{r}_{0}\right)+\mu_{0} \omega^{2} \mathbf{S}\left(\mathbf{r}_{0}, \mathbf{r}_{0}\right) \mathbf{p}\right]$, with $\boldsymbol{\alpha}(\omega)$ the polarizability tensor of the particle and $\mathbf{S}=\mathbf{G}-\mathbf{G}_{0}, \mathbf{G}_{0}$ being the Green tensor of the homogeneous medium of refractive index $n$ that surrounds the particle. 
In order to derive an upper bound that is independent of the absorber, we calculate the maximum of $P_{a}=f(\mathbf{p})$. In a complex medium composed of passive materials, the quantity $\omega \mathbf{u}^{\dagger}$ gu gives the total electromagnetic power emitted by the electric-dipole source $\mathbf{u}$ (see Supporting Information). Therefore, $\mathbf{u}^{\dagger} \mathbf{g u} \geqslant 0$ whatever the vector $\mathbf{u}$ and $\mathbf{g}$ is a semi-definite positive matrix. We now use this important property to derive the maximum of $P_{a}=f(\mathbf{p})$. For that purpose, we have to rewrite the dissipated power under the form $P_{a}=-\omega \mathbf{u}^{\dagger} \mathbf{g u}+A$, where $A$ is independent of $\mathbf{p}$. A few algebraic manipulations of Eq. (1) lead to (details can be found in (see Supporting Information))

$$
P_{a}=-\omega\left(\mathbf{p}^{\dagger}+\frac{i}{4} \mathbf{E}_{b}^{\dagger} \mathbf{g}^{-1}\right) \mathbf{g}\left(\mathbf{p}-\frac{i}{4} \mathbf{g}^{-1} \mathbf{E}_{b}\right)+\frac{\omega}{16} \mathbf{E}_{b}^{\dagger} \mathbf{g}^{-1} \mathbf{E}_{b}
$$

Now the dissipated power takes the form $P_{a}=-\omega \mathbf{u}^{\dagger} \mathbf{g u}+A$, with $\mathbf{u}=\mathbf{p}-\frac{i}{4} \mathbf{g}^{-1} \mathbf{E}_{b}$ and $A=\frac{\omega}{16} \mathbf{E}_{b}^{\dagger} \mathbf{g}^{-1} \mathbf{E}_{b}$. Since $\mathbf{g}$ is a semi-definite positive matrix, we know that $-\omega\left(\mathbf{p}^{\dagger}+\right.$ $\left.\frac{i}{4} \mathbf{E}_{b}^{\dagger} \mathbf{g}^{-1}\right) \mathbf{g}\left(\mathbf{p}-\frac{i}{4} \mathbf{g}^{-1} \mathbf{E}_{b}\right) \leqslant 0$. This readily leads to an upper bound for the absorption, which is independent of the absorber and depends only on the environment and the illumination, $P_{a} \leqslant P_{a}^{\max }$, with

$$
P_{a}^{\max }=\frac{\omega}{16} \mathbf{E}_{b}^{\dagger} \mathbf{g}^{-1} \mathbf{E}_{b}
$$

According to Eq. (2), the upper bound is reached for an optimal dipole $\mathbf{p}_{\mathrm{opt}}=\frac{i}{4} \mathbf{g}^{-1} \mathbf{E}_{b}$. The optimal polarizability that yields the maximum absorption is then (see Supporting Information)

$$
\boldsymbol{\alpha}_{\mathrm{opt}}(\omega)=\frac{c^{2}}{\omega^{2}}\left[\mathbf{S}^{*}\left(\mathbf{r}_{0}, \mathbf{r}_{0}\right)-i \frac{\omega n}{3 \pi c} \mathbf{I}\right]^{-1}
$$

with $\mathbf{S}^{*}$ the conjugate of $\mathbf{S}$. Any other polarizability in the same environment necessarily absorbs less light. Equation (4) can be seen as a vectorial generalization of the usual scalar impedance-matching concept ${ }^{32,35}$.

We define the absorption efficiency $\eta_{a}=P_{a} / P_{\text {inc }}$ as the fraction of the incident power 
that is absorbed inside the particle. The maximum absorption efficiency is

$$
\eta_{a}^{\max }=\frac{\omega}{16} \frac{\mathbf{E}_{b}^{\dagger} \mathbf{g}^{-1} \mathbf{E}_{b}}{P_{\text {inc }}}
$$

If the incident field is a plane wave, we rather define a maximum absorption cross-section by normalizing $P_{a}^{\max }$ with the incident Poynting vector $\frac{1}{2} \varepsilon_{0} c n\left|\mathbf{E}_{\mathrm{inc}}\right|^{2}$,

$$
\sigma_{a}^{\max }=\frac{3 \lambda^{2}}{8 \pi n^{2}} g_{0} \frac{\mathbf{E}_{b}^{\dagger} \mathbf{g}^{-1} \mathbf{E}_{b}}{\left|\mathbf{E}_{\mathrm{inc}}\right|^{2}},
$$

where $g_{0} \mathbf{I} \equiv \frac{1}{2} \mu_{0} \omega^{2} \operatorname{Im}\left[\mathbf{G}_{0}\left(\mathbf{r}_{0}, \mathbf{r}_{0}\right)\right]=\omega^{3} n /\left(12 \pi \varepsilon_{0} c^{3}\right) \mathbf{I}$. Note that, in a homogeneous environment, $\mathbf{g}=g_{0} \mathbf{I}, \mathbf{E}_{b}=\mathbf{E}_{\text {inc }}$, and we recover the usual result $\sigma_{a}^{\max }=3 \lambda^{2} /\left(8 \pi n^{2}\right)$.

Equations (3)-(6) form the central result of this article. They deserve a few comments before we illustrate their consequences on a couple of examples. The upper bound is independent of the absorber; it solely depends on the antenna and the incident field. Thus, Eqs. (5) and (6) provide meaningful figures of merit for comparing the ability of different systems to enhance absorption. These novel figures of merit result from an interplay between the local field $\mathbf{E}_{b}$ provided by the sole antenna and the imaginary part of its Green tensor $\mathbf{g}$.

In the case where the vectorial nature of the electromagnetic field can be neglected, the general upper bound can be replaced by an approximate form. Let us assume that the field $\mathbf{E}_{b}$ and the Green tensor $\mathbf{g}$ are dominated by a single component, the $z$ component. Within this scalar approximation, Eq. (6) reduces to

$$
\sigma_{a}^{\max } \approx \frac{3 \lambda^{2}}{8 \pi n^{2}} \frac{\left|E_{b z}\right|^{2}}{\left|E_{\mathrm{inc}}\right|^{2}} \frac{g_{0}}{g_{z z}}=\frac{3 \lambda^{2}}{8 \pi n^{2}} \frac{\text { Intensity enh. }}{\text { LDOS enh. }}
$$

The upper bound appears to be the ratio between the intensity enhancement $\left|E_{b z}\right|^{2} /\left|E_{\text {inc }}\right|^{2}$ and the LDOS enhancement $g_{z z} / g_{0}$. The fact that a LDOS enhancement is detrimental can be intuitively understood as follows: a larger LDOS increases the radiation of the induced dipole, i.e., it increases the scattering at the expense of absorption.

It can be shown using reciprocity that Eq. (7) is equivalent to $\sigma_{a}^{\max }=G_{a}(\theta, \phi) \lambda^{2} /\left(4 \pi n^{2}\right)$, 
with $G_{a}$ the antenna gain (see Supporting Information). The upper bound derived with antenna theory is thus a particular (scalar) case of the general result in Eq. (6). Two equivalent points of view can be adopted. They provide different conclusions and it is interesting to consider both of them. Equation (7) evidences that antennas supporting hot spots are a good choice if, and only if, the formation of hot spots is not concomitant with a large LDOS. On the other hand, the antenna gain is the product of the radiative efficiency and the directivity, $G_{a}(\theta, \phi)=\eta_{r} D(\theta, \phi)^{32}$. This second point of view underlines that (i) directional structures perform better and (ii) dielectric objects $\left(\eta_{r}=1\right)$ are better choices than plasmonic structures with $\eta_{r}<1$.

Let us illustrate the theory with a few examples. First, we study a plasmonic dimer nanoresonator and a Yagi-Uda antenna made of silicon nanospheres. Both can be treated in the scalar approximation. Secondly, we consider structures that can only be studied with a fully vectorial formalism: silicon nanospheres in a L-shape configuration and a SOI ridge waveguide.

In the case of a single-mode antenna, LDOS and field enhancement are not independent since both are driven by the excitation of an eigenmode ${ }^{36}$. Both are resonant with the same spectral profile and increasing one necessarily enhances the other. Thus, quite counterintuitively, the value of the upper bound in Eq. (7) is not correlated to the presence of hot spots. To illustrate this conclusion, let us consider a dimer made of two gold nanorods illuminated by a plane wave. Only the dipole mode at $\lambda=3 \mu \mathrm{m}$ is excited. Figure 2(a) displays the spectra of the maximum absorption cross-section and the intensity enhancement at the gap center: $\left|\mathbf{E}_{b}\right|^{2} /\left|\mathbf{E}_{\text {inc }}\right|^{2}$ is resonant whereas $\sigma_{a}^{\max }$ is not. Figure 2(b) shows the variation of $\left|\mathbf{E}_{b}\right|^{2} /\left|\mathbf{E}_{\text {inc }}\right|^{2}$ and $\sigma_{a}^{\max }$ with $w$. As $w$ is varied, the cylinder length is tuned to keep the resonance fixed at $\lambda=3 \mu \mathrm{m}$ so that all the antennas are at resonance. The maximum absorption cross-section only weakly varies while the field enhancement strongly decreases. It is noteworthy that, since $\sigma_{a}^{\max }<3 \lambda^{2} /\left(8 \pi n^{2}\right)$, any absorber in the gap of this plasmonic dimer absorbs less than an optimal absorber in a homogeneous medium. 

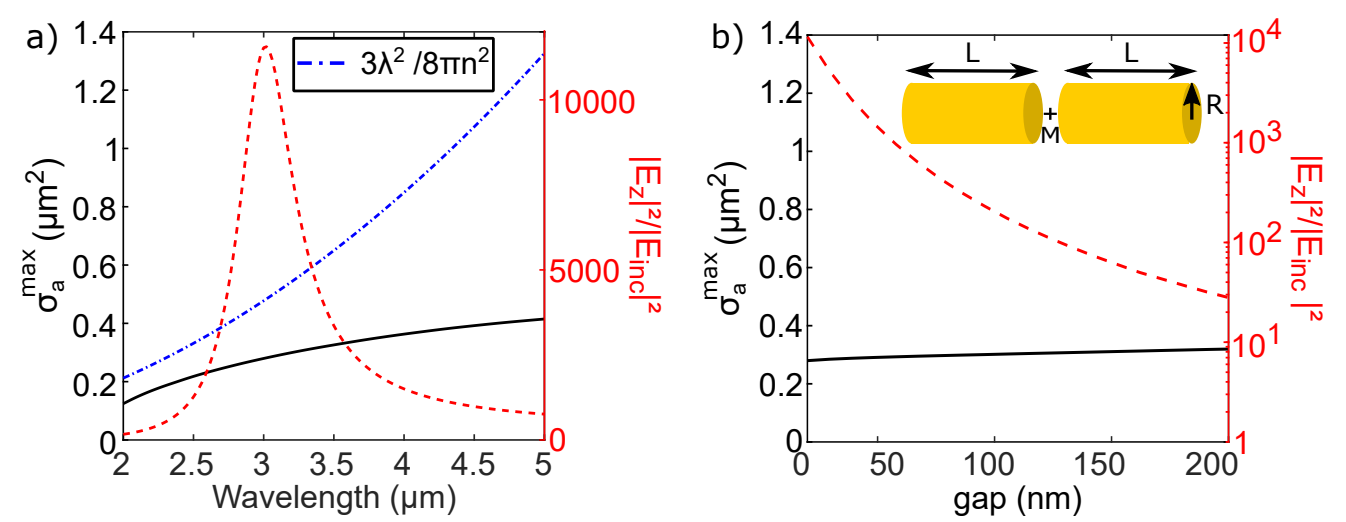

FIG. 2: Nanoantennas in the scalar approximation. Dimer composed of two gold cylinders illuminated by a normal-incident plane wave polarized along the cylinder axis. The point $M\left(\mathbf{r}_{0}\right)$ is the gap center. (a) Spectra of the maximum absorption cross-section $\sigma_{a}^{\max }$ (left axis, black curve) and the intensity enhancement (right axis, dashed red curve) for $L=414 \mathrm{~nm}, R=$ $15 \mathrm{~nm}$, and $w=20 \mathrm{~nm}$. (b) Maximum absorption cross-section and intensity enhancement as a function of the gap width $w$ (the cylinder length is tuned to keep the resonance fixed at $\lambda=3 \mu \mathrm{m})$. Calculations are performed with an aperiodic Fourier modal method dedicated to body-of-revolution objects ${ }^{37}$. The gold permittivity is given in (see Supporting Information). The antenna is embedded in a medium of refractive index $n=1.5$.

Since the upper bound in the scalar case is proportional to the radiative efficiency $\eta_{r}$ and the directivity, we switch to a Yagi-Uda antenna made of silicon nanospheres, which is known to be directional ${ }^{38}$. We keep a plane-wave illumination and a wavelength of $\lambda=3 \mu \mathrm{m}$. Thus, silicon is transparent and $\eta_{r}=1$. The maximum absorption cross-section $\sigma_{a}^{\max }$ is represented on Fig. 3(a) as a function of the incident angle $\theta$ for $\mathbf{r}_{0}$ located in between the reflector and the first director. The incident plane wave is either TM (black curve) or TE (red curve) polarized. For $\theta=0^{\circ}, \sigma_{a}^{\max }$ is one order of magnitude larger than $3 \lambda^{2} /\left(8 \pi n^{2}\right)$. This evidences that a dielectric directional antenna can provide larger absorption enhancements than a plasmonic antenna supporting hot spots. Note that, for a fixed geometry, the upper bound changes with the incident angle, whereas the optimal polarizability given by Eq. (4) is constant. Once the polarizability has been chosen, one is sure to reach the upper bound whatever the incident field.

Before considering fully vectorial examples, we discuss the possibility to reach the upper bound on the Yagi-Uda example. The optimal polarizability corresponds for instance to a 

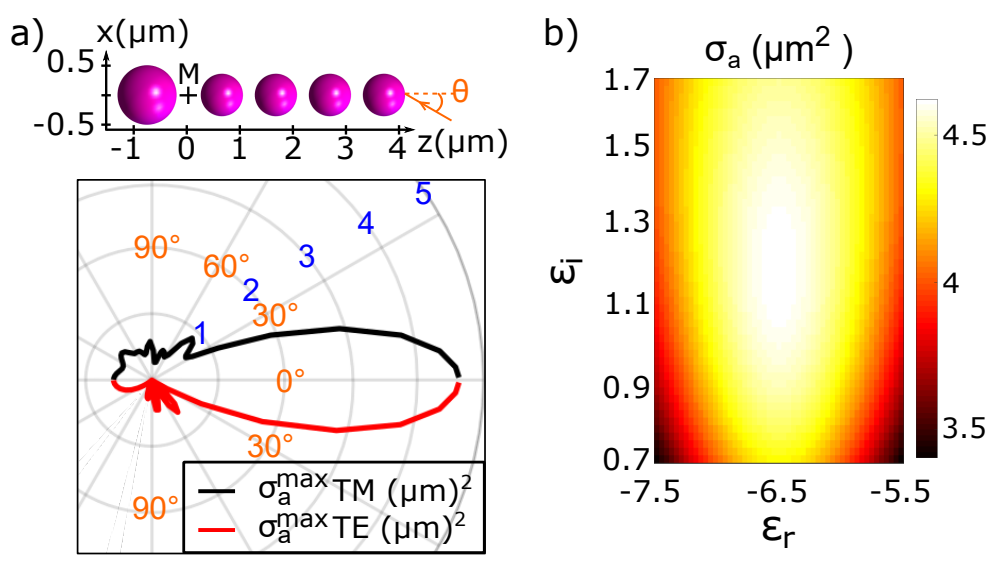

FIG. 3: Nanoantennas in the scalar approximation. Yagi-Uda antenna composed of silicon spheres (refractive index 3.436). The reflector (radius $498 \mathrm{~nm}$ ) is separated from the first director by $700 \mathrm{~nm}$. The four directors (radius $382 \mathrm{~nm}$ ) are equally spaced by $250 \mathrm{~nm}$. The point $M\left(\mathbf{r}_{0}\right)$ is the center between the reflector and the first director. (a) Maximum absorption cross-section $\sigma_{a}^{\max }$ at $\lambda=3 \mu \mathrm{m}$ as a function of the incident angle $\theta$ for a TM (black curve) or TE (red curve) polarized plane wave. (b) Absorption cross-section of an absorbing nanosphere of radius $176 \mathrm{~nm}$ inserted at $\mathbf{r}_{0}$. The real and imaginary parts of the absorber permittivity are varied around the optimal value $\varepsilon_{\mathrm{opt}}=-6.46+1.22 \mathrm{i}$. Calculations are performed with a rigorous multipole method ${ }^{38,39}$. The antenna is embedded in a medium of refractive index $n=1.5$.

sphere of radius $176 \mathrm{~nm}$ filled with a material of relative permittivity $\varepsilon_{\mathrm{opt}}=-6.46+1.22 i$; the relation between the electric-dipole polarizability, the sphere radius, and the permittivity has been rigorously calculated with Mie theory. Such a permittivity at $\lambda=3 \mu \mathrm{m}$ can be obtained with highly-doped semiconductor nanocrystals ${ }^{40,41}$. We have checked with a rigorous numerical method that inserting this nanosphere in the Yagi-Uda antenna yields an absorption that is indeed equal to the upper bound (see Supporting Information). We have also tested the robustness of the optimal polarizability. Figure 3(b) shows the absorption cross-section at normal incidence in TM polarization obtained by varying the absorber permittivity around the optimum. Since the cross-section varies smoothly around the maximum, we have a good flexibility on the choice of the permittivity.

Let us now consider a L-shape antenna made of three silicon spheres, see Figs. 4(a)-(b). Such a structure cannot be described in the scalar approximation, especially for positions $\mathbf{r}_{0}$ outside the symmetry planes. In that case, Eq. (7) is not valid. The system can only be 

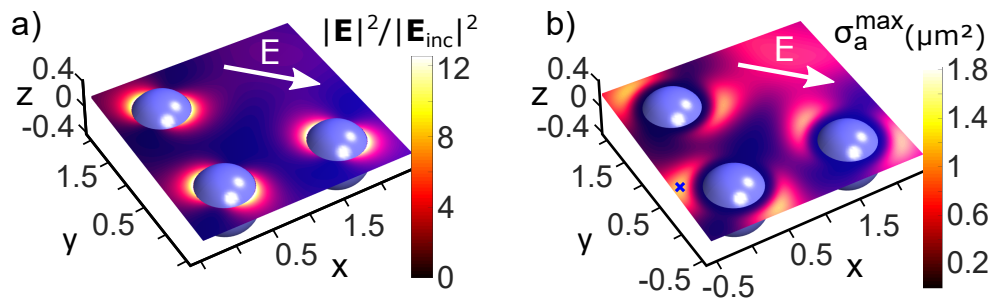

FIG. 4: Beyond scalar approximation. L-shape antenna made of three silicon spheres (radius $430 \mathrm{~nm}$ ) equally spaced by $1.54 \mu \mathrm{m}$ and embedded in a medium of index $n=1.5$ at $\lambda=3 \mu \mathrm{m}$. (a) Intensity enhancement and (b) maximum absorption cross-section as a function of the position $\mathbf{r}_{0}$ in the $(x, y)$ plane for $z=232 \mathrm{~nm}$. The map of the intensity enhancement is clearly different from the spatial distribution of the maximum absorption cross-section. Calculations are performed with a rigorous multipole method $^{38,39}$.

characterized by the vectorial upper bound of Eq. (6). The latter depends on the absorber position and it is important, for a given nanoantenna, to evaluate where the particle could reach the best absorption. Figures 4(a)-(b) show respectively the spatial distributions of the intensity enhancement and the maximum absorption cross-section in the $(x, y)$ plane for $z=232 \mathrm{~nm}$. The antenna is illuminated from the bottom by a plane wave propagating along the $z$ axis and polarized linearly along the white arrow. The blue cross in Fig. 4(b) marks the position where the upper bound is maximum. It does not correspond to the maximum of $\left|\mathbf{E}_{b}\right|^{2} /\left|\mathbf{E}_{\text {inc }}\right|^{2}$, which evidences, once again, that seeking hot spots is not sufficient to maximize the absorption.

Let us study a last example where the incident field is not a plane wave. It allows us to evidence the generality of the absorption upper bound that can be used in a variety of photonic structures. We consider an absorber located above a SOI ridge waveguide. In this integrated configuration, the incident field is a guided mode. By applying Eq. (5), we have calculated the maximum absorption efficiency above the waveguide, see Fig. 5. The absorber is moved along the horizontal white line located $20 \mathrm{~nm}$ above the waveguide; the incident field is either the fundamental quasi-TE mode (solid black curve) or the quasi-TM mode (dashed red curve). The latter provides an absorption efficiency of almost 50\%, even for an absorber located outside the waveguide core.

In conclusion, we derived an upper bound for the problem of absorption by a dipolar 

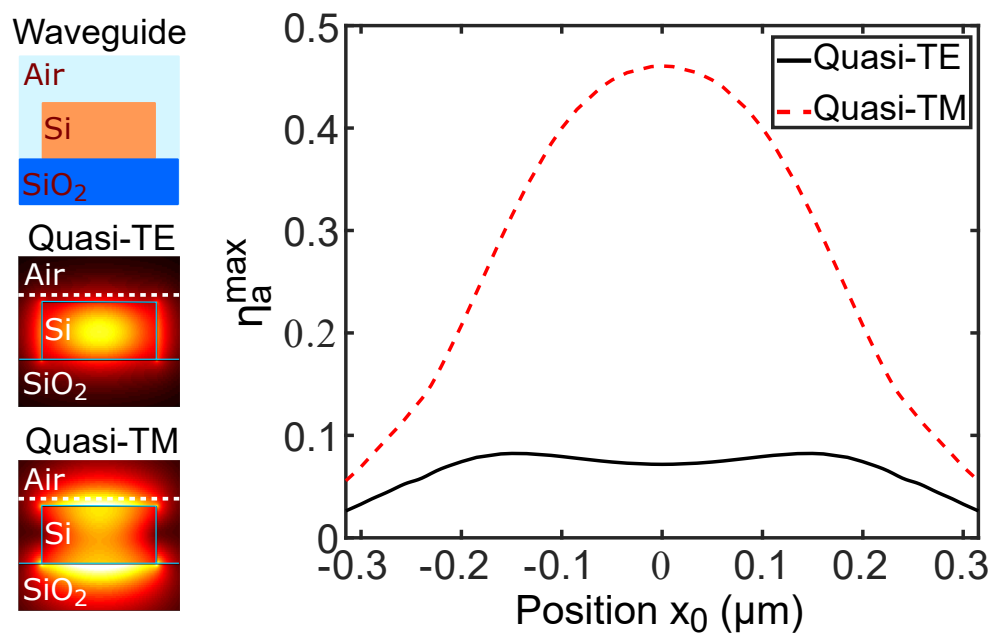

FIG. 5: Beyond scalar approximation and plane-wave illumination. SOI ridge waveguide at $\lambda=1.5 \mu \mathrm{m}$. The silicon ridge $(n=3.5$, width $500 \mathrm{~nm}$, thickness $250 \mathrm{~nm})$ lies over a silica substrate $(n=1.44)$. Maximum absorption efficiency as a function of the position along the dashed white line located $20 \mathrm{~nm}$ above the waveguide for the fundamental quasi-TE mode (solid black curve) and the quasi-TM mode (dashed red curve). The insets give the electric-field intensity distributions of both modes.

absorber in a complex environment, homogeneous or not. The derivation relies on a vectorial formalism and is valid for any environment and any illumination. Since it decouples the environment from the absorber, the upper bound provides a meaningful figure of merit for comparing the intrinsic ability of different structures to enhance absorption in a nanovolume. Moreover, it allows seeking, for one given structure, the optimal position for the absorber. In the scalar approximation, the relevant parameter is not the field enhancement but the ratio between field enhancement and LDOS. Thus, although placing an absorber in a hotspot of a plasmonic structure can increase the absorption ${ }^{10}$, plasmonic antennas supporting hot spots are not necessarily the best candidates to reach high absorption enhancements. Larger enhancements can be achieved with dielectric directional antennas. In cases where the scalar approximation is not valid or the illumination is not a plane wave, the situation is more complex and our general upper bound is the good figure of merit to characterize the problem. Since it relies on electromagnetic calculations (field enhancement and Green tensor) that are nowadays performed routinely, we think that this work opens new avenues for understanding and optimizing absorption in subwavelength volumes inserted in complex media. Thanks to 
its generality, this concept should benefit various applications in several spectral domains: SEIRA, photovoltaics, thermal emission or nanochemistry. This work could also serve as a building block for the study of multipolar and/or multiple absorbers.

\section{Supporting Information Available}

The following file is available free of charge:

- Supporting Information for Publication.pdf: we provide in this document some additional elements concerning the numerical calculations and the derivations of the upper bound and of the optimal polarizability presented in the main text.

\section{References}

(1) Beer, A. Bestimmung der Absorption des rothen Lichts in farbigen Flussigkeiten. Annalen der Physik und Chemie 1852, 86, 78-88.

(2) Einstein, A. Uber einen die Erzeugung und Verwandlung des Lichtes betreffenden heuristischen Gesichtspunkt. Annalen der Physik 1905, 17, 132-148.

(3) Atwater, H. A.; Polman, A. Plasmonics for improved photovoltaic devices. Nature Mater. 2010, 9, 205-213.

(4) Vynck, K.; Burresi, M.; Riboli, F.; Wiersma, D. S. Photon management in twodimensional disordered media. Nature Mater. 2012, 11, 1017-1022.

(5) Mubeen, S.; Hernandez-Sosa, G.; Moses, D.; Lee, J.; Moskovits, M. Plasmonic Photosensitization of a Wide Band Gap Semiconductor: Converting Plasmons to Charge Carriers. Nano Letters 2011, 11, 5548-5552, PMID: 22040462. 
(6) Baldassarre, L.; Sakat, E.; Frigerio, J.; Samarelli, A.; Gallacher, K.; Calandrini, E.; Isella, G.; Paul, D. J.; Ortolani, M.; Biagioni, P. Midinfrared Plasmon-Enhanced Spectroscopy with Germanium Antennas on Silicon Substrates. Nano Letters 2015, 15, $7225-7231$.

(7) Neubrech, F.; Huck, C.; Weber, K.; Pucci, A.; Giessen, H. Surface-Enhanced Infrared Spectroscopy Using Resonant Nanoantennas. Chem. Rev. 2017, 117, 5110-5145.

(8) Dong, L.; Yang, X.; Zhang, C.; Cerjan, B.; Zhou, L.; Tseng, M. L.; Zhang, Y.; Alabastri, A.; Nordlander, P.; Halas, N. J. Nanogapped Au Antennas for Ultrasensitive Surface-Enhanced Infrared Absorption Spectroscopy. Nano Letters 2017, 17, 57685774, PMID: 28787169.

(9) Jin, M.; Belkin, M. A. Infrared Vibrational Spectroscopy of Functionalized Atomic Force Microscope Probes using Resonantly Enhanced Infrared Photoexpansion Nanospectroscopy. Small Methods 2019, 3, 1900018.

(10) Sakat, E.; Wojszvzyk, L.; Hugonin, J.-P.; Besbes, M.; Sauvan, C.; Greffet, J.-J. Enhancing thermal radiation with nanoantennas to create infrared sources with high modulation rates. Optica 2018, 5, 175-179.

(11) Greffet, J.-J.; Bouchon, P.; Brucoli, G.; Marquier, F. m. c. Light Emission by Nonequilibrium Bodies: Local Kirchhoff Law. Phys. Rev. X 2018, 8, 021008.

(12) Christopher, P.; Ingram, D. B.; Linic, S. Enhancing Photochemical Activity of Semiconductor Nanoparticles with Optically Active Ag Nanostructures: Photochemistry Mediated by Ag Surface Plasmons. The Journal of Physical Chemistry C 2010, 114, $9173-9177$.

(13) Carlson, M. T.; Green, A. J.; Richardson, H. H. Superheating Water by CW Excitation of Gold Nanodots. Nano Letters 2012, 12, 1534-1537, PMID: 22313363. 
(14) Baffou, G.; Quidant, R. Thermo-plasmonics: using metallic nanostructures as nanosources of heat. Laser Photonics Rev. 2013, 7, 171-187.

(15) Tretyakov, S. Maximizing Absorption and Scattering by Dipole Particles. Plasmonics 2014, 9, 935âĂ

(16) Fleury, R.; Soric, J.; Alù, A. Physical bounds on absorption and scattering for cloaked sensors. Phys. Rev. B 2014, 89, 045122.

(17) Miller, O. D.; Hsu, C. W.; Reid, M. T. H.; Qiu, W.; DeLacy, B. G.; Joannopoulos, J. D.; Soljačić, M.; Johnson, S. G. Fundamental Limits to Extinction by Metallic Nanoparticles. Phys. Rev. Lett. 2014, 112, 123903.

(18) Grigoriev, V.; Bonod, N.; Wenger, J.; Stout, B. Optimizing Nanoparticle Designs for Ideal Absorption of Light. ACS Photonics 2015, 2, 263-270.

(19) Colom, R.; Devilez, A.; Bonod, N.; Stout, B. Optimal interactions of light with magnetic and electric resonant particles. Phys. Rev. B 2016, 93, 045427.

(20) Miller, O. D.; Polimeridis, A. G.; Reid, M. T. H.; Hsu, C. W.; DeLacy, B. G.; Joannopoulos, J. D.; Soljačić, M.; Johnson, S. G. Fundamental limits to optical response in absorptive systems. Opt. Express 2016, 24, 3329-3364.

(21) Ivanenko, Y.; Gustafsson, M.; Nordebo, S. Optical theorems and physical bounds on absorption in lossy media. Opt. Express 2019, 27, 34323-34342.

(22) Hugonin, J.-P.; Besbes, M.; Ben-Abdallah, P. Fundamental limits for light absorption and scattering induced by cooperative electromagnetic interactions. Phys. Rev. B 2015, 91, 180202 .

(23) Castanie, E.; Vincent, R.; Pierrat, R.; Carminati, R. Absorption by an Optical Dipole Antenna in a Structured Environment. International Journal of Optics 2012, 452047. 
(24) Sentenac, A.; Chaumet, P. C.; Leuchs, G. Total absorption of light by a nanoparticle: an electromagnetic sink in the optical regime. Opt. Lett. 2013, 38, 818-820.

(25) Jackson, J. D. Classical Electrodynamics; J. Wiley and Sons: New York, 1974.

(26) Novotny, L.; Hecht, B. Principles of Nano-Optics, 2nd ed.; Cambridge University Press: Cambridge, 2006.

(27) Purcell, E. M. Spontaneous emission probabilities at radio frequencies. Phys. Rev. 1946, 69,681 .

(28) Goy, P.; Raimond, J.-M.; Gross, M.; Haroche, S. Observation of Cavity-Enhanced Single-Atom Spontaneous Emission. Phys. Rev. Lett. 1983, 50, 1903-1906.

(29) Sauvan, C.; Hugonin, J.-P.; Maksymov, I. S.; Lalanne, P. Theory of the Spontaneous Optical Emission of Nanosize Photonic and Plasmon Resonators. Phys. Rev. Lett. 2013, $110,237401$.

(30) Seok, T. J.; Jamshidi, A.; Kim, M.; Dhuey, S.; Lakhani, A.; Choo, H.; Schuck, P. J.; Cabrini, S.; Schwartzberg, A. M.; Bokor, J.; Yablonovitch, E.; Wu, M. C. Radiation Engineering of Optical Antennas for Maximum Field Enhancement. Nano Letters 2011, 11, 2606-2610, PMID: 21648393.

(31) Metzger, B.; Hentschel, M.; Schumacher, T.; Lippitz, M.; Ye, X.; Murray, C. B.; Knabe, B.; Buse, K.; Giessen, H. Doubling the Efficiency of Third Harmonic Generation by Positioning ITO Nanocrystals into the Hot-Spot of Plasmonic Gap-Antennas. Nano Letters 2014, 14, 2867-2872, PMID: 24730433.

(32) Balanis, C. A. Antenna theory: Analysis and design, 2nd ed.; J. Wiley and Sons: New York, 1997.

(33) Andersen, J. B. Absorption Efficiency of Receiving Antennas. IEEE Transactions on Antennas and Propagation 2005, 53, 2843 - 289. 
(34) Ra'di, Y.; Tretyakov, S. A. Balanced and optimal bianisotropic particles: maximizing power extracted from electromagnetic fields. New Journal of Physics 2013, 15, 053008.

(35) Greffet, J.-J.; Laroche, M.; Marquier, F. Impedance of a Nanoantenna and a Single Quantum Emitter. Phys. Rev. Lett. 2010, 105, 117701.

(36) Lalanne, P.; Yan, W.; Vynck, K.; Sauvan, C.; Hugonin, J.-P. Light interaction with photonic and plasmonic resonances. Laser Photonics Rev. 2018, 12, 1700113.

(37) Bigourdan, F.; Hugonin, J.-P.; Lalanne, P. Aperiodic-Fourier modal method for analysis of body-of-revolution photonic structures. J. Opt. Soc. Am. A 2014, 31, 1303-1311.

(38) Stout, B.; Devilez, A.; Rolly, B.; Bonod, N. Multipole methods for nanoantennas design: applications to Yagi-Uda configurations. J. Opt. Soc. Am. B 2011, 28, 1213-1223.

(39) Stout, B.; Auger, J.-C.; Lafait, J. A transfer matrix approach to local field calculations in multiple-scattering problems. Journal of Modern Optics 2002, 49, 2129-2152.

(40) Mendelsberg, R. J.; Garcia, G.; Milliron, D. J. Extracting reliable electronic properties from transmission spectra of indium tin oxide thin films and nanocrystal films by careful application of the Drude theory. Journal of Applied Physics 2012, 111, 063515.

(41) Mendelsberg, R. J.; Garcia, G.; Li, H.; Manna, L.; Milliron, D. J. Understanding the Plasmon Resonance in Ensembles of Degenerately Doped Semiconductor Nanocrystals. The Journal of Physical Chemistry C 2012, 116, 12226-12231. 